\title{
LA LUZ COMO YUGO, LA VERDAD COMO NO-OCULTAMIENTO Y EL ALMA COMO ASPIRACIÓN UN COMENTARIO A: MARTIN HEIDEGGER, DE LA ESENCIA DE LA VERDAD
}

LIGHT AS A YOKE, TRUTH AS UNCONCEALMENT AND SOUL AS ASPIRATION. COMMENTS TO: MARTIN HEIDEGGER, ON THE ESSENCE OF TRUTH

\author{
Alberto Ciria \\ Munich (Alemania)
}

Recibido: 20-05-2014

Aceptado: 10-08-2014

Resumen: En este artículo se presentan las lecciones que bajo el título "De la esencia de la verdad" Heidegger pronunció en 1931/32 sobre dos textos platónicos. La luz se concibe como unión de la verdad como el estar al descubierto de lo ente (tratado en la parábola de la caverna) y el alma como aspiración al ser (tratada en el Teeteto).

Palabras-clave: Heidegger, Platón, luz, alma, ser, verdad

\begin{abstract}
The article deals with the readings given by Heidegger in 1931/32 of two texts by Plato under the title of "On the Essence of Truth". Light is conceived as being a union of Truth as the being uncovered of entity (in the Allegory of the Cave) and the soul as the aspiration of being (in the Theaetetus).

Key-words: Heidegger, Plato, light, soul, being, truth
\end{abstract}

\section{La luz como foco y como yugo. Lo recogido en la luz como vi- sible y como vidente. (La luna)}

Las lecciones De la esencia de la verdad, que Heidegger pronunció en el semestre de invierno de 1931/32 en la Universidad de Friburgo, y que el editor subtituló "Sobre la parábola de la caverna y el Teeteto de Platón", son una reflexión sobre la luz, en forma de dos comentarios a sendos textos platónicos. 
La luz es, pues, el centro de toda la obra. Pero en el $§ 3$ se dice que "luz", Licht, es un término equívoco. Con esta equivocidad se corresponde una dualidad de términos tanto en griego ( $\pi \tilde{e} \rho$ y $\varphi \tilde{u} \varsigma$ ) como en latín (lux y lumen).

Luz significa, por un lado, la fuente de luz: el foco, que es puntual.

Luz significa, por otro lado, el espacio iluminado, la zona donde hay luz, expandida a partir de su foco: la claridad, die Helle, que es un ámbito.

Un ámbito de luminosidad se llama también un "claro", Lichtung.

A partir de la etimología de la palabra Helle, en $\S 6$ se dice que la claridad es lo que permite pasar a través: lo transparente, lo permeable, lo transitable. Lichten significa iluminar y también significa aclarar, despejar, de modo que ahora se puede pasar a través. Pero lo transparente, lo permeable o lo transitable sólo es posible como una referencia, siempre es lo transparente-para (la visión), lo permeable-para (el agua) o lo transitable-para (un hombre). Que la claridad, en su carácter esencialmente referencial, sea el ámbito de luminosidad que se expande a partir de la luz como foco puntual, significa que esos dos elementos: donde puede transitarse y lo que puede transitar, son capacitados para su respectivo carácter transicional por la luz, que en esta capacitación respectiva los une, como un yugo que los unce (§ 13).

En la imagen simbólica, el sol es la fuente de la luz, que "capacita" a lo visible para ser visible y al vidente para ver, de modo que ambos, cada uno a su modo, son hechos con arreglo al sol, es decir, son heliomórficos (§ 13).

Con arreglo a estos dos que son uncidos por la luz, capacitándolas ésta para su respectivo carácter transicional, y haciéndolos así "heliomórficos", el libro tiene dos partes:

La primera parte (§§ 1-19), un comentario a la parábola de la caverna, versa sobre la "visibilidad", Sichtbarkeit.

La segunda parte ( $\S \S 20-46)$, un comentario al Teeteto, versa sobre la "videncialidad", Sichtsamkeit.

Visibilidad y videncialidad sólo son posibles en su relación mutua, en su unción bajo el mismo yugo: la luz que los posibilita a ambos.

A esa "unción" o pertenencia mutua obedece que, tanto en alemán como en castellano, términos como Wahrnehmung o Ansicht, "percepción" o "visión", designen tanto lo visto como el acto de ver (\$24). Esta misma dualidad de significados aparece, finalmente, en los propios términos griegos de íća y de $\delta$ ó $\xi \alpha$ (§37).

\section{La visibilidad de las sombras. (Marte)}

En la parábola de la caverna, la luz como foco y como yugo es el sol, y la claridad como ámbito transitable es todo el recorrido desde las cadenas, que sólo permiten ver sombras, hasta el exterior de la caverna, desde donde se puede contemplar primero la luz expandida desde el sol, y finalmente el propio sol. 
La luz como yugo, la verdad como no-ocultamiento y el alma como aspiración.

Un comentario a: Martin Heidegger, De la esencia de la verdad

Pero las sombras no son sólo el negativo de lo visible, sino que también ellas mismas son visibles, es decir, también las sombras quedan recogidas, como un primer estadio, dentro del recorrido a través del ámbito de claridad: en la oscuridad completa no existen las sombras.

Así como las sombras no son la negación o el negativo de lo visible,

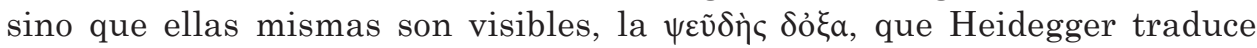
como verkehrte Ansicht, "visión invertida", no es un no-conocimiento, sino una forma límite de conocimiento. En correspondencia con $\S 3$, dedicado al primer estadio de la parábola de la caverna, la vida en las cadenas y en las sombras, el quinto y último capítulo de las lecciones (§§ 39-46) está dedicado a la "visión invertida".

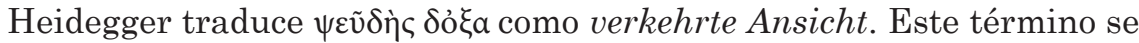
traduciría en general como "opinión falsa", o mejor aún, "visión errónea" de un asunto. En $\S 46$ dice Heidegger que "opinión”, Meinung, es ya una forma derivada, "reconfigurada", de la dóxa. En esta edición se ha traducido verkehrte

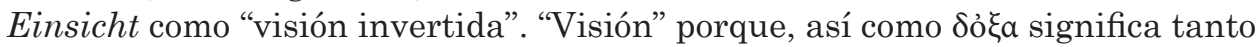
el aspecto, la imagen o la apariencia que algo ofrece por sí mismo, como la reputación en la que es tenido -y en un sentido extremo, la gloria- (§ 37), Ansicht, igual que "visión", significa tanto "opinión", como lo visto, como el acto de ver (por ejemplo en la forma ansichtig werden, cfr. § 46) (ibíd.). E "invertida" porque una opinión equivocada no es un mero no-conocer, ni una visión errónea es un mero no-ver, ni tampoco lo visto erróneamente deja de ser visto, es decir, deja de mostrarse, sino que, en todo caso, mostrándose se oculta, queda oculto tras un mostrar... un mostrar girado, vuelto al revés, invertido, que no oculta meramente escondiendo, sino que oculta mostrando otra cosa a la que hace pasar por aquella otra que esconde. Heidegger lo ilustra con el caso del "pseudónimo" (§ 18), mediante el cual se oculta el nombre verdadero dando a ver otro. De esta manera, igual que ha "salvado" a las sombras incluyéndolas entre lo visible, Heidegger puede hablar también de la "salvación del fenómeno

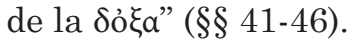

La verdad como no-ocultamiento no es entonces la verdad como rectitud o corrección cuyo opuesto excluyente sea la falsedad o la incorrección, sino la verdad que, por consistir en un desencubrimiento, no existe fuera de una relación esencial y una pertenencia al encubrimiento. Porque, de este modo, el encubrimiento queda incorporado a la verdad, también el rostro girado, y la visión invertida, quedan integrados dentro de lo cognoscible y del conocimiento aun como formas límites.

THÉMATA. Revista de Filosofía, Nº50 julio-diciembre (2014) pp.: 215-227

doi: 10.12795/themata.2014.i50.10 


\section{Captación y mirada, lo ente y el alma. (Mercurio)}

De esta manera, a diferencia del error y de la falsedad, la inversión, el girar o dar la vuelta significa de suyo una dualidad de caras o de rostros (§38), una doblez del aspecto, a la que, por su parte, se dirige la dóxa como visión en una bifurcación, Gabelung. A esta bifurcación están dedicados los tres últimos parágrafos (§§ 44-46).

Que la $\delta$ ỏ $\xi a$ como "visión" se dirija en una bifurcación al "doble rostro"

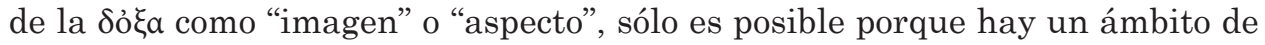
claridad donde todo ente puede salir por vez primera al encuentro ofreciendo una imagen (que en su doblez puede estar invertida) y al cual el alma está

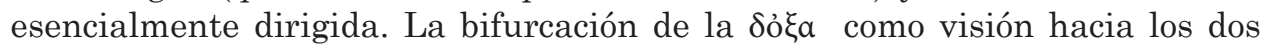
rostros de la $\delta$ ỏ $\alpha$ como "imagen"o "aspecto" es posible sobre la base de una bifurcación más original: la bifurcación entre la "captación", Erfassung, de lo ente, y la "mirada esencial", Wesensblick, del hombre al ser, sólo a cuya luz puede salir al encuentro lo ente. En esa bifurcación más original llegamos a conocer cuál es la esencia de la verdad:

"[La esencia de la verdad] consiste en que la existencia del hombre tiene que tener de entrada la mirada esencial para el ser, tiene que haberse liberado para la íśca, la única a cuya luz lo ente puede salir al encuentro sin encubrimiento. Mirándolo de otro modo: el ser tiene que mantenerse de entrada en la aspiración para que lo ente nos pueda ser confiado.

¿Pero con qué topamos con el fenómeno de la mirada esencial y de la aspiración al ser? Con el fenómeno que constituye la estructura esencial de la $\delta o ̉ \xi \alpha$ y que nosotros llamamos la bifurcación." (§ 46)

El término "bifurcación", Gabelung, designa originalmente un camino que se desdobla o se ramifica en dos direcciones ahorcajadas, de modo que hay que optar por una o por otra. Ya en su comentario a la parábola de la caverna Heidegger había dicho que lo esencial de la parábola no son los estadios, sino las transiciones de un estadio a otro. Pero, como "esencia de la verdad", la bifurcación no es una alternativa, sino dos ramales que se recorren simultáneamente, o dos "púas" que se clavan simultáneamente. Ambos ramales son simultáneamente transitables porque ambos se dirigen hacia lo mismo convergiendo en él, y ambos ramales se dirigen convergiendo hacia lo mismo porque, en realidad, un ramal, el ahorcajado, no es más que el marco del otro, el recto. Pero ya hemos visto que lo transitable no es sólo transitable-hacia, sino que es siempre transitable-para. Pues bien: el "hacia" es lo ente, y el "para" es el alma.

La "transitabilidad" o "permeabilidad" del ser, o dicho en términos de luz, su "transparencia", hace visible. Lo visible sólo es visible en medio de lo transparente. La "visibilidad" del ente, que el ente pueda ofrecer un aspecto tomando dicho aspecto en relación con aquello que permite que aparezca, 
La luz como yugo, la verdad como no-ocultamiento y el alma como aspiración.

Un comentario a: Martin Heidegger, De la esencia de la verdad

eso gracias a lo cual es visible en el sentido de que puede hacerse ver, es la verdad, que por eso, a su vez en términos de luz, se concibe como no-ocultamiento, $\grave{\alpha} \lambda \dot{n} \theta \varepsilon \iota \alpha$.

Por su parte, el alma no es un sujeto que disponga de la facultad de referirse al ser transitando, sino que el alma no existe fuera de la referencia al ser, o mejor dicho, el alma es la referencia al ser en forma de transitar. El alma no transita, sino que el alma es el transitar. El ser es lo transitable, y el alma es el estar transitando. En esa referencia es posible la "percepción" o "conocimiento", que es el tema central del diálogo Teeteto. Por eso Heidegger puede a su vez hablar del conocimiento como una "conducta" o un "comportamiento", Verhalten, porque el comportamiento es esencialmente referencial. Todo comportamiento es hacia o respecto de algo, en este caso, lo ente: Verhalten zum Seienden. El conocimiento no es primaria y esencialmente conocimiento del conocimiento, ni conocimiento en sí mismo, sino que el conocimiento es origi-

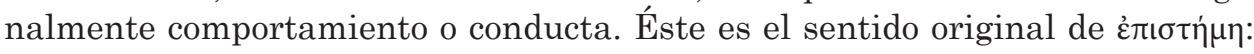
no "entender algo", sino "entender de algo" (sich verstehen auf, sich auskennen in), por ejemplo entender de la confección de zapatos, entender de agricultura, de ganadería, o entender de cómo llevar una guerra. $(§ 20)$

El alma no percibe ni conoce el ser "a través de" facultades, sino que, en la relación del hombre con el ser, el alma es el propio "a través".

El "a través" es siempre un "a través hacia", pero también es siempre un "a través de" o un "a través en".

Transitabilidad es penetrabilidad. Para la mente en el sentido más amplio, para el espíritu, para el alma, penetrabilidad es inteligibilidad (Durchdringung es, por ejemplo, un término central en Fichte). Inteligibilidad es logos. El alma no sólo atraviesa el logos, sino que el alma es, para el hombre, el atravesarlo. "A través", en griego, se dice dia-. A través del logos, es diálogo. El diálogo es, así, el elemento del que está hecha el alma. El pensar sobre el alma, sobre el ser, sobre el foco que los unce uno a otro, se formula como un diálogo porque, primero, el alma es, según su esencia, diálogo.

\section{Alma como aspiración. (Júpiter)}

Para describir que el alma es el propio "a través" o el propio tránsito en el ser hacia lo ente, Heidegger emplea términos como Streben, Bestreben, Anstreben, que -matizando uno u otro aspecto- significan en general "tender". Pero sobre todo emplea un término de la misma familia que será central para todo su comentario al Teeteto: Erstreben o Er-streben, y más en concreto Seinserstrebnis, que en esta edición se ha traducido como "aspiración al ser".

Para conservar toda la multiplicidad de términos que emplea Heidegger, en esta edición se ha traducido Streben, que es el término más básico, como "tendencia"; Bestreben, como "esforzarse por"; das Bestrebte, que el propio 
Heidegger (en $\S 30$ d) caracteriza como el objeto de la tendencia a tener, se ha traducido como "lo pretendido". ¿Pero cómo traducir entonces Erstreben, Erstrebnis (Seinserstrebnis) y Er-streben?

La palabra aparece por vez primera como designación del tercer paso del despliegue de las referencias del percibir, que -en cuatro pasos- ocupa el tercer capítulo de la segunda parte. La primera caracterización del Erstrebnis se encuentra en $\S 29$ :

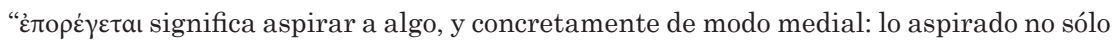
es algo que el alma ansía, sino que es aspirado para ella misma y sólo para ella.”

En $\S 30$ se dice:

“Qué significa “aspirar”? Ésta es aquí la pregunta importante. ¿Qué significa en general esta relación del hombre con el ser en el sentido de eso que llamamos "aspiración al ser"? Percibir es un "tener ante si" que asume algo dado. Pero si, al percibir algo, la relación del alma con el ser debe ser un tender, entonces, en una relación de tendencia tal, lo percibido no puede ser tenido."

\section{Y finalmente, en $\S 31$ :}

"Tal tender no tiende a la posesión de lo pretendido, sino a que algo pretendido permanezca mantenido en el tender, para que el que tiende, desde lo pretendido y hacia sí mismo, pueda llegar hasta sí mismo. Tal tender, qua aspirar, sería entonces un tender auténtico, en el que el sí mismo tendente no tiende alejándose de sí, sino regresando hacia sí, para, tendiendo de este modo, aspirar a sí pretendiendo, es decir, para ganarse a sí mismo en el tender. Lo pretendido y lo aspirado no coinciden, pero ciertamente van juntos en la esencia de este tender, es más, este tender es lo que constituye la unidad.”

"Lo pretendido y lo aspirado no coinciden." Lo pretendido, das Bestrebte, es el objeto que se busca en el tender, aquello a lo que se tiende. Lo aspirado, das Erstrebte, no es el objeto de la aspiración, sino... quien aspira, el aspirante: por eso se habla de un "aspirar a sí" (§ 31). ¿Qué es una aspiración, a diferencia de una pretensión? No se aspira a un objeto, a la posesión de un objeto. A lo mejor se aspira a un título o a un cargo, a la posesión de un título o de un cargo. ¿Pero qué significa aquí "poseer un cargo"? No significa "tenerlo" como una posesión; significa "ocuparlo", estar en él. ¿Y qué significa "ocupar"? Significa convertirlo en espacio propio, pero de modo que uno no se ha apropiado del cargo, sino que se ha hecho a sí mismo apropiado para él. Lo aspirado no es el cargo como propiedad, sino uno mismo como estando en el cargo en tanto que apropiado para él. Es decir, en último término, lo aspirado es... el aspirante mismo. Por eso dice Heidegger que, en la aspiración, el tendente "no se aleja de sí", sino que "regresa hacia sí" y "se gana a sí mismo".

Ésta es la diferencia entre ambicionar y aspirar, como dos modos del tender. Ambicionar significa tender a hacer propio lo pretendido. Aspirar significa tender a hacer-se propio de lo pretendido, en el sentido de apropiado 
La luz como yugo, la verdad como no-ocultamiento y el alma como aspiración.

Un comentario a: Martin Heidegger, De la esencia de la verdad

para ello. En $\S 30$ se decía que, porque la percepción es una tendencia como aspiración, "lo percibido no puede ser tenido".

Porque la relación del alma con el ser es de aspiración, y aspirar significa tender a hacerse apropiado para lo pretendido, la pregunta por la esencia de la verdad, la pregunta por el ser, es decir, la filosofía misma, exige del hombre una transformación. (§15)

Pero por mucho que en el aspirar lo aspirado sea el aspirante mismo en tanto que busca hacerse apropiado, existe un término de la aspiración, pues de otro modo ella no sería una tendencia ni, en general, una relación. Para enfatizar esta dimensión transitiva o este carácter terminal que pese a todo tiene toda aspiración, Heidegger escribe en ocasiones el término separando el prefijo: Er-streben. Er- es un prefijo que significa finalización, término, consecución. De modo correspondiente, se ha traducido Er-streben como "aspirar pretendiendo", cuyo significado ha explicado Heidegger en el texto ya citado: "aspirar a sí pretendiendo: [...] lo pretendido y lo aspirado no coinciden, pero ciertamente van juntos en la esencia de este tender, es más, este tender es lo que constituye la unidad."

Ser uno mismo apropiado para algo, que es a lo que se aspira en la aspiración, se dice también ser idóneo. En su explicación más detenida del alma como "aspiración al ser" (§ 32), Heidegger menciona la idoneidad/no-idoneidad (Tauglichkeit/Untauglichkeit), junto con la belleza/fealdad, como referencia del ser, en el sentido de que siempre se es idóneo para algo. Pero ser apropiado, o hacer apropiado, se dice también estar capacitado o capacitar, en el sentido de haber recibido o de dar potestad -en el sentido más general- para algo: Ermächtigen. Ya antes se ha dicho que la luz capacita para ser visible y para ver, para la visibilidad y para la videncialidad. "A esto que capacita, Platón lo llama: ảjäóv. Nosotros lo traducimos como el bien. El significado auténtico y propio de ảjatóv se refiere a aquello que vale para algo y que hace que otras cosas valgan para algo, con las que se puede hacer algo. [...] No tiene nada del significado de lo moralmente bueno.” (§ 14) El alma como "aspiración al ser", que es la noción central del comentario al Teeteto, está así referida a la idea del bien, representada por el sol como fuente de luz, que es la noción central del comentario a la parábola de la caverna. Aspirar significa tender a hacerse apropiado, a valer para algo, a ser idóneo o "bueno" para algo, en el sentido mencionado de "bien": tender a estar capacitado. El bien es entonces, según esta definición, lo que posibilita la aspiración y le da sentido. O diciéndolo con un término filosófico posterior, el bien es el transcendental de la aspiración. Por eso dice Heidegger: "La capacitación es el límite de la filosofía." (§ 14)

Las sombras y el sol como foco de luz son los límites de lo visible: no hay visión por debajo de las sombras ni más allá del sol. La "visión invertida" y el conocimiento del bien son los límites del conocimiento, y por tanto de la

THÉMATA. Revista de Filosofía, Nº50 julio-diciembre (2014) pp.: 215-227

doi: 10.12795/themata.2014.i50.10 
filosofía: no hay conocimiento inferior a la "visión invertida" ni superior a la idea del bien.

El alma no dispone de una facultad para acceder al ser, sino que el propio alma es el "a través" del ser: éste es el sentido más elemental del alma como aspiración. Pero, aparte de basar la traducción de Seinserstrebnis como designación del alma en las explicaciones que da Heidegger, hay un motivo etimológico para traducir dicha expresión como "aspiración al ser".

"Alma" procede del latín anima, que significa "aire", "aliento", "hálito", igual que "espíritu" procede del latín spiritus, que significa "soplo" o "viento".

Anima, a su vez, procede del griego ánemos, "viento", al igual que spiritus procede del griego physa, "fuelle", y del verbo physáo, "soplar". (Cfr. A. Ernout y A. Meillet, Dictionnaire étymologique de la langue latine: histoire des mots, París, Klincksieck, 1960).

Además de anima y spiritus, una tercera palabra, Pneuma, que sobre todo en el contexto de la filosofía estoica se suele traducir como "espíritu", también significa originalmente "soplo", "hálito", "respiración".

De la raíz spiritus procede en castellano toda la familia de palabras "respiración", "expiración", "espiración” y, finalmente, "aspiración”. Por tanto, al margen de la interpretación del alma como Seinserstrebnis, etimológicamente "alma" remite por sí misma a la "aspiración".

De esta comprensión del alma como un hálito proceden los diversos relatos religiosos, mitológicos, literarios y populares de la infusión de vida mediante un soplo y de la recepción de vida mediante una inspiración o de su recuperación mediante un beso (cfr. por ejemplo Gn 2, 7). Y esta comprensión es también vigente cuando el sentido de la infusión se invierte: "La corporalidad [...] sólo puede haberla inculcándola en un alma, y no al revés, que un alma se le insufle a un cuerpo." (§ 24)

Sin embargo, no he podido constatar una conexión etimológica entre spiritus y pyr, "luz".

\section{Tener presente y hacer presente: una bifurcación ahorcajada. (Venus)}

Lo visible sólo puede salirnos al paso en medio de lo transparente. Nosotros percibimos o captamos (wahrnehmen, erfassen) lo visible sólo porque, de entrada, estamos referidos ya a lo transparente. Sólo dentro de la referencia a lo transparente es posible percibir lo visible y captar lo ente. Todos estamos dentro de esa referencia a lo transparente, en algún lugar entre las cadenas y el sitio desde donde se puede contemplar directamente el sol como foco de luz, que son los dos límites del ámbito de luminosidad, del claro, y por tanto también de la visión. Todos estamos en el claro. A lo mejor los animales también. Pero no todos, ni constantemente, captamos el ente en cuanto ente, igual que 
La luz como yugo, la verdad como no-ocultamiento y el alma como aspiración.

Un comentario a: Martin Heidegger, De la esencia de la verdad

no todos, ni constantemente, captamos las sombras como sombras. El ente sólo es posible captarlo en cuanto tal, la sombra sólo es posible tomarla como sombra, haciendo presente, simultáneamente con la captación, la referencia al ser o a la transparencia.

Heidegger distingue entre gegenwärtigen y vergegenwärtigen: "tener presente" y "hacer presente" (§ 42-44). Tener presente es el estar dado inmediatamente en presencia nuestra lo ente en su comparecencia corporal. Hacer presente es traer a presencia nuestra lo que no está compareciendo inmediatamente.

Hay tantos modos de hacer presente como modos hay de no estar compareciendo inmediatamente a nuestra presencia. Un modo de no estar compareciendo inmediatamente es haber sido olvidado: $\lambda \dot{n} \theta \eta$ (a partir del cual se forma la palabra ả $\lambda \dot{n} \theta \varepsilon ı \alpha, \S 18$ ). Otro modo de no estar compareciendo es estar oculto. Y otro modo de no comparecer es, finalmente, ser transparente: el ámbito donde toda cosa puede comparecer inmediatamente, no comparece a su vez inmediatamente. Por eso inicialmente está oculto y olvidado.

En $\S 44$ Heidegger habla de la "bifurcación" entre "tener presente" y "hacer presente", que él esquematiza gráficamente al final del último parágrafo. La bifurcación tiene dos ramales, uno recto y el otro ahorcajado. Es posible recorrerlos simultáneamente a ambos, porque también ambos se dirigen hacia lo ente, y ambos se dirigen hacia lo ente porque, como se ha dicho, el ramal recto está como enmarcado en el ahorcajado. El ramal recto es la "captación", Erfassung, de lo visible o de lo ente por estar inmediatamente dado a la vista o al conocimiento. La captación es de lo visible o de lo ente, pero inicialmente no es de lo visible en cuanto visible ni de lo ente en cuanto ente. El ramal ahorcajado es la referencia al ser, la aspiración al ser o la mirada esencial. Sólo poniendo en relación la captación con la mirada esencial se capta lo visible en cuanto visible y lo ente en cuanto ente, y esa relación es, justamente, la bifurcación, pero precisamente la bifurcación en ahorcajamiento de dos líneas convergentes.

\section{El suelo y la bóveda de la caverna: otra imagen de ahorcaja- miento. (Saturno)}

En la parábola de la caverna, tras el arrancamiento de las cadenas y el ascenso al exterior, al tercer estadio de la contemplación primero de la luz del sol y finalmente del sol como fuente de luz, le sucede un cuarto estadio de regreso y de descenso al interior de la caverna, a las sombras, después de haber visto la luz del sol, cuya contemplación el liberado trae consigo, quizá en forma de un recuerdo. El liberado regresa a la caverna a liberar a los otros: él mismo es un liberador. ¿Pero en qué consiste esta liberación? No es una liberación de las sombras, sino una liberación de las cadenas. ¿Y qué son las cadenas? Lo que fuerza a tomar las sombras como las cosas reales, o dicho de otro modo, lo

THÉMATA. Revista de Filosofía, №50 julio-diciembre (2014) pp.: 215-227 doi: 10.12795/themata.2014.i50.10 
que impide ver las sombras como sombras. La liberación no consiste en dejar de ver las sombras: las sombras no merecen que se las deje de ver, porque no son la negación de lo visible, sino que ellas mismas son visibles. Las sombras merecen ser vistas, y merecen ser vistas como sombras, pero eso sólo es posible poniéndolas en relación con una luz cuya contemplación uno trae consigo y hace presente recordándola simultáneamente al contemplar las sombras.

Un ramal ahorcajado, un arco, se traza con una línea que primero asciende y luego desciende regresando. El cuarto estadio de la parábola de la caverna, el descenso de regreso a las sombras llevando consigo y haciendo presente el recuerdo de la luz del sol, se corresponde con el ramal ahorcajado de la bifurcación: captar lo directamente visible haciendo presente simultáneamente lo único en medio de lo cual lo visible es visible, es decir, lo que capacita a lo visible para ser visible y, por tanto, al vidente para ver, y que, por consiguiente, no es ello mismo visible ni vidente: lo transparente. La captación tiene presente lo visible, y la mirada esencial hace presente lo transparente.

Esta "bifurcación", Gabelung, entre tener presente y hacer presente, o entre captación y mirada esencial, se corresponde con la diferencia entre ente y ser, porque éstos son, respectivamente, los referentes de aquéllas. Sin embargo, en estas lecciones "diferencia" no es todavía un término específico central.

Esta misma diferencia entre tener presente y hacer presente, entre ente y ser, entre captación y mirada esencial, entre "a través hacia" y "a través de", se corresponde a su vez con la diferencia entre pretender y aspirar. Y la bifurcación como articulación de los susodichos binomios en tanto que caminos simultáneamente transitables, se corresponde entonces con la forma lingüística Er-streben, un "aspirar pretendiendo" expresado en una palabra única pero desdoblada y articulada mediante un guión.

En el estilo de redacción de Heidegger es muy frecuente el uso de guiones para enlazar palabras, y también para separar en las palabras el prefijo de su raíz. En el caso de Er-streben, la separación mediante guión de prefijo y raíz no pretende enfatizar el prefijo para llamar de esta forma la atención sobre una modulación del significado de la palabra, es decir, no pretende acentuar la transitividad del pretender, el hecho de que el pretender tiene un objeto directo. El guión no enfatiza el significado de uno de los componentes de la palabra, sino que dentro de ella cobra un significado propio, que exhibe además plásticamente: su significado como separación y como unión, en este caso, la bifurcación del transitar en aspiración y pretensión. Porque la bifurcación es tanto la separación en dos ramales como su unión, en el sentido de que los ramales resultan de una división del camino, pero si no estuvieran unidos, juntados justamente en su punto de división, tampoco serían ramales uno de otro, sino dos caminos independientes.

Con la bifurcación del Er-streben en aspirar y pretender se corresponde el desdoblamiento del ver en mirada esencial y en captación, con los que se corresponde la diferencia entre ser y ente. 
La luz como yugo, la verdad como no-ocultamiento y el alma como aspiración.

Un comentario a: Martin Heidegger, De la esencia de la verdad

Pero esta diferencia -como muestra plásticamente la parábola de la caverna- no es primera, sino que previamente la diferencia ha sido capacitada para serlo. Lo que capacita es, según su sentido etimológico original, el bien. Si falta el foco, entonces no hay diferencia entre la luz y las tinieblas. El foco es el "haya luz", la cual, por ser "buena", se separa de la oscuridad (Gn 1, 3-4). La diferencia entre dos sólo es posible gracias a un tercero.

Hay la diferencia entre lo que comparece (lo ente) o lo que captamos (las sombras y las cosas), y el claro dentro del cual aquello puede comparecer y al cual el alma está referido. Pero el claro no es más que la expansión de la luz desde su foco. Lo transparente hace visible a lo visible, pero el foco de luz hace transparente a lo transparente. Aquí hay tres niveles, que se corresponden con los tres primeros estadios de la parábola de la caverna y que son reunidos de modo peculiar en el cuarto: 1) lo directamente visible o captable, lo ente, que en la parábola de la caverna son las sombras y las cosas y en el esquema de la bifurcación se corresponde con el ramal recto; 2) el ámbito de claridad, transparencia, transitabilidad y permeabilidad, donde lo visible puede ser visto y lo ente puede ser encontrado, que en la parábola de la caverna es recorrido en el ascenso y en el esquema de la bifurcación se corresponde con el ramal ahorcajado; y 3) el punto desde donde se expande el ámbito de claridad, que a su vez capacita a lo transitable para ser transitable: en la parábola de la caverna es el sol o la idea del bien, y en el esquema de la bifurcación es lo que tensa y sostiene el arco en tensión del ramal ahorcajado, con su peculiar forma de yugo.

En el "Proemio" de Parménides, conocimiento y opinión, verdad y reputación, la verdad de cada uno de nosotros que en muchas dimensiones nos excede y esa representación que tenemos de nosotros mismos y a la que nos aferramos, en la que nos encerramos y en la que vivimos, también se describen como vías, es decir, como transiciones, pero como transiciones excluyentes; ciertamente ofrecen asimismo una bifurcación, pero es una bifurcación de vías progresivamente divergentes desde un punto de arranque común, representado como una alternativa. En la parábola platónica, existe la diferencia entre conocimiento y opinión (dóxa como visión), pero no en forma de dos vías excluyentes y divergentes, sino de dos vías convergentes: existe una bifurcación en dos vías, pero en dos vías que en su término convergen, ofreciendo la forma de un "ahorcajamiento". ¿Y por qué son convergentes en su término? Porque, si ambas vías son recorridas realmente, en ningún momento se separan.

Los ramales del ahorcajamiento no representan entonces una alternativa excluyente. Se puede no recorrer el ramal ahorcajado si se omite el hacer presente -cuando tomamos las sombras como los entes-, pero hacer presente en ningún caso excluye el tener presente, sino que, justamente, sólo puede hacerse a partir de él, igual que una condición esencialmente oculta de posibilidad sólo puede sacarse de su ocultamiento desde la patencia de lo posibilitado. $\mathrm{E}$ igual que hacer presente no excluye tener presente, lo tenido presente

THÉMATA. Revista de Filosofía, Nº50 julio-diciembre (2014) pp.: 215-227

doi: 10.12795/themata.2014.i50.10 
tampoco es un negativo de lo hecho presente. La opinión no es la negación del conocimiento, no es su negativo, sino su sombra. ¿Y qué es una sombra, según nos muestra plásticamente la parábola de la caverna? Una sombra es una proyección. ¿Y qué es una proyección? Una proyección es un desplazamiento del aspecto que lo absuelve del ente que originalmente lo ofrece, pero de forma que, en tanto que aspecto que jamás deja de serlo, se sigue ofreciendo a sí mismo. Por ser un desplazamiento tal, una proyección es siempre una reducción, aunque espacialmente pueda ser mayor o incluso más nítida en cuanto al aspecto. Pero porque el aspecto consiste esencialmente en ofrecerse, la sombra siempre es esencialmente visible, y tomando la visión como metáfora del conocimiento, cognoscible. Y en efecto la conocemos si la tomamos precisamente como proyección, es decir, poniéndola a ella, que la tenemos presente, en referencia al ente del cual ella ha sido desplazada, un ente que no tenemos presente pero que, en esa referencia, es hecho presente.

Por el contrario, la sombra la desconocemos -no la vemos como sombra- si la tomamos por el ente mismo -como hacen los encadenados-, lo cual nos sucede cuando nos atenemos sólo a lo que tenemos presente y omitimos el hacer presente. ¿Quién es el encadenado? El que no puede hacer presente, sino que está condenado a atenerse únicamente a lo que tiene presente.

La sombra es un aspecto proyectado, y la esencia del aspecto es ofrecerse. Y ofrecerse significa hacerse ser tenido presente. Cuando caemos en el engaño de tomar la sombra como el ente, no estamos violentando a la sombra, sino ateniéndonos a lo que ella es, pero únicamente a eso. Por eso, tomar la sombra como el ente, siendo eso un engaño, no es una equivocación, ni tampoco es una falsedad. La sombra sólo la conocemos como tal y en lo que ella es si la conocemos respecto de algo que no es tenido en ella, pero que hace posible que ella sea tenida.

Así se puede decir que, según la parábola de la caverna, la opinión es una sombra o una proyección del conocimiento, y por eso, sin ser por sí misma verdadera, tampoco es sin más falsa. Ésta es otra forma de explicar lo que antes se ha llamado el "doble rostro" de la $\delta$ ó $\xi$.

Toda esta historia de la caverna, a lo largo de sus cuatro estadios, no es más que una parábola de una manera de contemplación, que consiste en tener presente a la luz de lo hecho presente; y si la contemplación la tomamos a su vez como una metáfora del conocimiento, toda la narración no es más que la parábola de un modo de conocer.

Aparte del doble camino de ascenso y de regreso en descenso a las sombras, en la parábola de la caverna hay otra imagen del ramal ahorcajado como marco de la visión: es el propio techo abovedado de la caverna, tendido entre la sombra y la salida a la luz del sol, pero que a su vez, según la concepción antigua, representa el universo como bóveda ( $\$ 5)$. Lo que sostiene la tensión de la bóveda celeste teniéndola alzada y permitiéndole, capacitándola para dar 
La luz como yugo, la verdad como no-ocultamiento y el alma como aspiración.

Un comentario a: Martin Heidegger, De la esencia de la verdad

cabida en sí a los entes con los que topamos y a nosotros mismos, es el punto de la "clave" de bóveda, más allá de la cual no podemos ver: el sol como imagen del bien, entendido a su vez como capacitación, que define así un límite de la filosofía. La capacitación es la tensión entre el aspirante y el cargo.

La clave de bóveda es el punto donde la bóveda entera se reúne: es la juntura de la bóveda. Y también es el punto desde donde la bóveda entera se expande: es el foco de la bóveda. La clave capacita a la bóveda para ser lo que ella es. Pero en tanto que punto, no está directamente presente en la bóveda, sino bajo la forma de un límite.

\section{Lo que falta: la filosofía como nostalgia. (Descanso final)}

Hacer presente lo que no está compareciendo directamente, recordar lo olvidado, desencubrir lo oculto: tales movimientos no son primeros, sino que sólo pueden ejercerse respecto de algo que previamente falta, ya sea en el modo de estar ausente, de haber sido olvidado, de estar oculto o de estar perdido. Dicho de otro modo, hacer presente, recordar o desencubrir sólo son posibles como una "intervención", Einsatz, que según las circunstancias podrá percibirse como una "acometida" o "ataque", Angriff, o incluso como una "violencia", Gewalt, que es lo que experimenta el encadenado cuando se le fuerza a volver la cabeza.

La intervención no es forzosamente una negación. En todo caso, la negación es un modo de la intervención. La verdad como no-ocultamiento no significa negación del ocultamiento, sino intervenir sobre el ocultamiento, que de este modo queda incorporado esencial y positivamente a la verdad. La verdad como no-ocultamiento significa que la verdad es la intervención sobre una falta, ya sea ésta una ausencia, un olvido, un ocultamiento o una pérdida. La verdad nace de un echar en falta. El impulso a la verdad, la búsqueda de la verdad, el amor a la verdad, vienen de un echar en falta. En Los conceptos fundamentales de la metafísica: mundo, finitud, soledad (GA 29/30 § 2b), citando unas palabras de Novalis, Heidegger había definido la filosofía como una "nostalgia".

En resumen: De la esencia de la verdad es una reflexión sobre la luz concebida como un yugo que unce en su pertenencia mutua, capacitándolas, la verdad como no-ocultamiento de lo ente (tratada en la parábola de la caverna) y el alma como aspiración al ser (tratada en el Teeteto).

THÉMATA. Revista de Filosofía, №50 julio-diciembre (2014) pp.: 215-227

doi: 10.12795/themata.2014.i50.10 
